\title{
EL VALOR DE LA ARITMÉTICA MERCANTIL \\ EN EL DESARROLLO DE LAS TÉCNICAS \\ MERCANTILES MEDIEVALES*
}

\author{
Betsabé Caunedo del Potro \\ Universidad Autónoma de Madrid
}

\section{ResUmen}

El objetivo de este trabajo es fijarnos en la rápida difusión de la aritmética mercantil por Europa Occidental en la Baja Edad Media. Los manuales de la nueva disciplina debían formar profesionales preparados para afrontar los desafíos y transformaciones que el comercio y la banca exigían. La formación era causa del éxito profesional. Lectura, escritura y cálculo resultaban imprescindibles.

Palabras clave: expansión medieval, desarrollo del comercio, formación mercantil, disciplinas básicas: escritura, lectura y cálculo, instrumentos de trabajo.

\section{THE ROLE OF MERCANTILE ARITHMETIC IN THE DEVELOPMENT \\ OF MEDIEVAL MERCANTILE TECHNIQUES}

\section{Abstract}

The aim of this essay is to highlight the fast dissemination of mercantile arithmetic throughout Western Europe in the Late Middle Ages. The manuals of the new discipline had to prepare men capable of addressing the challenges and transformations that trade and banking demanded. Training was the cause of professional success. Reading, writing and calculation skills were essential.

Keywords: Medieval expansion, trade development, mercantile education and training, basic subjects: reading, writing and calculus, working tools. 


\section{INTRODUCCIÓN}

En este trabajo me gustaría reflexionar sobre la rápida difusión de las aritméticas mercantiles por Europa Occidental desde el siglo XII relacionando esta veloz propagación con el espectacular desarrollo del comercio en esos años. Realmente creemos que fue una consecuencia natural y necesaria de la rápida expansión y desarrollo de la actividad comercial, pero a la vez vemos a este género emerger como protagonista irrefutable de unas novedosas técnicas mercantiles que garantizaron y respaldaron el crecimiento y progreso de la actividad en los años centrales de la Edad Media.

Una primera, rápida y quizá somera mirada nos lleva a explorar el Mediterráneo en los bulliciosos años de la Plena Edad Media. Vemos cómo a un lado y a otro de ese mar e incluso más allá, se intercambiaban mercancías muy diversas: esclavos, vino, aceite, sal, trigo, paños, especias, telas finas... Éstas se pesaban, medían y pagaban con pesos, medidas y monedas muy diferentes, acuñadas en distintos lugares, prestos a exigir multitud de gravámenes, cargas, aranceles, tasas o peajes... Un sinfín de pormenores, de particularidades, de detalles dificultaban extremadamente la actividad y hacían evidente la necesidad de moverse con orden y garantía. La escritura y la aritmética proporcionaron un buen apoyo y facilitaron «ese» orden, convirtiéndose en una poderosa razón para que arraigaran y triunfaran en las zonas de mayor proyección comercial.

Esa necesidad de orden puede ser una notable razón, pero no única. Vayamos más allá. Las técnicas mercantiles habían ido evolucionando desde el siglo XI. Primero pausadamente, después a mayor velocidad, se había ido recorriendo un largo camino de desarrollo y progreso, no exento de obstáculos, que permitió superar la excepcionalidad y discontinuidad del rudimentario comercio de los primeros ańos de la Edad Media. La definitiva sedentarización del mercader, la profesionalidad del servicio de transporte, las nuevas formas de asociación mercantil y de aportación de capital, el mayor uso de los sistemas de crédito, los nuevos modos de llevar la contabilidad... requerían que el mercader conociese mercados, rutas, instrumentos de cambio, medios de transporte o reglas aritméticas con las que efectuar diferentes operaciones y llevar con eficacia su nueva contabilidad ${ }^{1}$. Igualmente, esas nuevas formas de asociación situaron a algunos mercade-

* Este trabajo ha sido realizado en el marco del proyecto HAR2015-67619-P, Tecnología y Conocimiento en la Península Ibérica (siglos XIII-XVI), cofinanciado por el Ministerio de Economía y Competitividad y el Fondo Europeo de Desarrollo Regional.

1 Seguimos siendo deudores de los planteamientos de R. de Roover, «The commercial revolution of the thirteenth century». Bulletin of the Business Historical Society, vol. xvi (1942), pp. 34-39, $\mathrm{y}$ "The organization of trade», en The Cambridge Economic History of Europe, vol. III, Cambridge, University Press, 1965, pp. 42-118. Además, problemas como los endeudamientos de las monarquías, las pérdidas de créditos, las quiebras de bancos, las alteraciones monetarias sufridas durante la denominada "crisis del siglo xiv» habían alertado al mercader. C. Cipolla, El gobierno de la moneda. Ensayos de historia monetaria. Barcelona, Crítica, 1994, pp. 153-159. 
res como cabezas de sus compañías y gestores responsables ante sus "compañeros», sus socios, de los negocios que capitaneaban. Necesitaban una correspondencia mercantil y una buena red de información que asegurase el éxito de sus negocios o desviase su competencia. Ese éxito suponía beneficios que deberían ser repartidos. De nuevo unas reglas aritméticas básicas se dibujaban como imprescindibles. En ese ambiente, un aprendizaje determinado empezaba a perfilarse como razón del éxito empresarial a la vez que se consideraba imprescindible ${ }^{2}$.

Este aprendizaje tuvo un sólido componente práctico. Desde edades tempranas, encontramos a jóvenes llamados a ejercer la actividad comercial, acompañando a los más experimentados en la ejecución de sus labores y funciones ${ }^{3}$. Este aspecto práctico, muy valioso, sin duda, no fue el único. De modo simultáneo, los futuros mercaderes se iniciaban en una instrucción teórica, que cubría una serie de etapas y que comenzaba con la enseńanza de la lectura, la escritura y el cálculo. Leer, escribir y contar se perfilaron pronto como habilidades indispensables en el mundo mercantil. Se valoraron no por sí mismas, sino como herramientas de trabajo que permitían y facilitaban una mejor administración de los negocios y propiedades ${ }^{4}$. El mercader requería una formación instrumental para satisfacer unas necesidades básicas entre las que deberíamos volver a resaltar las siguientes: intercambiar noticias, establecer precios de compra y venta, calcular intereses, atender a la correspondencia de pesos y monedas, manejar distintos tipos de monedas en circulación, girar letras de cambio, expedir recibos, preparar balances... El contenido de su formación estaría totalmente adaptado a su realidad diaria. Los jóvenes transferían y aplicaban a su puesto de trabajo los aprendizajes y competencias alcanzados durante su formación. Esta perfecta adecuación entre teoría y práctica se esgrime como una de las claves del éxito alcanzado.

¿Cómo puede el principal de una compañía de comercio realizar sus funciones de dirigir, controlar y evaluar las actividades de su empresa si no recibe una información fecunda procedente de otros niveles de la organización? ¿Cómo

2 Hace ya varios años, Hilario Casado nos explicaba cómo la creación de instituciones y técnicas novedosas permitieron que las compañías castellanas redujesen costes y fuesen más eficaces y competitivas. Ello había favorecido el éxito y prosperidad del comercio castellano a fines de la Edad Media. H. Casado Alonso, «Comercio y nacimiento del Estado Moderno en Castilla (ss. XIV-XVI). Algunas reflexiones a la luz de nuevas corrientes de investigación internacional», en El Estado en la Baja Edad Media. Nuevas perspectivas metodológicas, Zaragoza, Universidad de Zaragoza, 1999, pp. 51-75.

${ }^{3}$ Ver al respecto mi trabajo «La formación y educación del mercader», en El Comercio en la Edad Media, XVI Semana de Estudios Medievales Nájera y Tricio 2005, Nájera, Instituto de Estudios Riojanos, pp. 417-454.

${ }^{4}$ Antonio Castillo Gómez nos explica cómo se trataba de un alfabetismo de cuño utilitario, práctico y profesional. Una alfabetización restringida, en la que el aprendizaje «no era un fin en sí mismo, sino consecuencia de un uso necesario o directamente útil». A. Castillo Gómez, Escrituras y escribientes. Práctica de la cultura escrita en una ciudad del Renacimiento. Las Palmas de Gran Canaria, Fundación de Enseñanza Superior a Distancia de Las Palmas de Gran Canaria, 1997, p. 271. 
puede notificar las decisiones a tomar? ¿Cómo comunicarse con sus factores? En este intercambio de noticias resultaba crucial saber utilizar unas adecuadas herramientas de comunicación, pues las informaciones debían resultar precisas, fidedignas y oportunas para los fines de la organización. Entre las diferentes formas de comunicación, el lenguaje escrito adquirió un enorme desarrollo, resultando la carta el medio de comunicación por excelencia. Fue el recurso adoptado para establecer contactos, llegar a acuerdos y realizar transacciones ${ }^{5}$. En estas misivas, en la transmisión de noticias, de informaciones e incluso de rumores o intuiciones se primaba la celeridad para no perder la ventaja de anticiparse en la toma de decisiones. Como sucede en la actualidad, también en los siglos XIv y xv la información constituía un poderoso recurso a la hora de obtener ventajas competitivas. Podemos resaltar como ejemplo el que en Brujas, a finales del siglo xv, no hubo reunión de la Nación Castellana en la que no se hiciese referencia, de un modo u otro, a la necesidad de noticias, a la llegada o a la tardanza de mensajeros o correos ${ }^{6}$, porque no sólo se debía enviar información, sino conseguir que se recibiese adecuadamente. El mantener una correcta comunicación escrita pronto se reveló como uno de los aspectos más importantes de una compañía. Las cartas ofrecían numerosas ventajas: reafirmaban la autoría al fijarse por escrito y firmarse; se suponían precisas, ya que se podían revisar y corregir antes de enviarse, y además las propuestas, tareas o funciones por ellas transmitidas podrían reformularse o ejecutarse. Se mostraron, desde el primer momento, como un pilar de apoyo fundamental en la toma de decisiones comerciales. No cabe duda de que podían facilitar información sobre aspectos críticos de vital importancia ${ }^{7}$.

Deberíamos continuar preguntándonos ¿cómo se pueden presentar cuentas a los diferentes socios y repartir beneficios entre los mismos si no se sabe efectuar unos mínimos cálculos? ¿Cómo era posible realizar un balance y presen-

5 En el trabajo elaborado en colaboración con M. SÁNCHEZ, "Cláusulas comerciales: ¿Acatamiento o trasgresión? El factor de negocios en la Europa Atlántica», en J.Á. Solórzano Telechea, B. Arizaga Bolumburu, L. Sicking (eds.), Diplomacia y Comercio en la Europa Atlántica Medieval, Logroño, Instituto de Estudios Riojanos, 2015, pp. 187-219 (en adelante "Cláusulas comerciales»), describimos cómo en ellas cabían todos los asuntos, desde la más simple operación comercial hasta las más delicadas operaciones diplomáticas. A la vez que señalamos como ejemplos notables de géneros especializados que surgieron de las cartas la emergencia de instrumentos financieros como letras de cambio, cartas de obligación, cartas de pago o crédito... destacamos la enorme importancia conferida por el Consulado de Burgos a las misivas.

${ }^{6}$ En «Cláusulas comerciales» comentamos también cómo en las reuniones del Consulado se leían públicamente las cartas que escribían los cónsules de Burgos e incluso cartas privadas con información de interés para toda la nación, pp. 196-197.

7 En «Cláusulas comerciales», p. 197, analizamos cómo el Consulado de Burgos dedicaba importantes recursos humanos y financieros para crear y mantener un sistema de correos rápido y efectivo para contar con una información fidedigna. Y cómo para adelantarse a la competencia no era suficiente utilizar los mismos canales que los demás mercaderes de la nación. Para ser el primero en dar la noticia el factor debe anticiparse y utilizar un servicio de correos propio y exclusivo de su compañía o acudir a viajeros o maestres en los que confiar las cartas que deben ser entregadas en puertos peninsulares. 
tar unas cuentas sin esos mínimos conocimientos? Y aunque existió un abismo entre el gran comercio internacional y el pequeńo comercio al detalle, todos los que practicaron cada una de esas modalidades, al igual que otros muchos sectores urbanos, fueron conscientes del valor de la lectoescritura y de la aritmética como condición de ascenso social en ese ámbito. Por ello, este tipo de instrucción se propagó. Unos y otros sintieron la imperiosa necesidad de formación, manifestaron un cierto culto a las letras, a las cifras, al manejo correcto de las mismas, también a las proporciones, a la exactitud..., propiciando un nuevo sistema de valores que lentamente acabaría imponiéndose ${ }^{8}$. La capacidad de leer, escribir y contar permitió claramente ascender o defender posiciones. Se reconocía y estimaba su valor, a la vez que se desaprobaba y rechazaba a quien no lo poseía? La formación empezaba a tener influencia en la promoción social. No cabe duda de que el modo de llevar los negocios conllevó nuevas demandas profesionales y nuevas exigencias, percibiéndose con claridad que una nueva economía exigía nuevas competencias. Y la formación, la adquisición de esas competencias, se mostró como un elemento clave para el desarrollo con éxito de la actividad de una organización mercantil. Resultó decisiva para generar buenos resultados que dependían cada vez más de unos conocimientos técnicos. Ser un buen profesional del comercio implicaba una buena formación. En este marco de crecimiento, en el que tomar ventaja requería capacitarse en nuevas habilidades, se difundieron desde Italia o a raíz de su iniciativa todas las innovaciones técnicas.

\section{MANUALES DE ÁBACO}

De todos es sabido que la península italiana capitaneó el flamante proceso de desarrollo comercial, especialmente la Toscana. Encontramos también a esta región a la vanguardia en el campo del aprendizaje comercial y de la disciplina matemática. Dispusieron para ello de unos manuales específicos-manuales de ábaco-y de una institución -las escuelas de ábaco-que garantizaban la instruc-

${ }^{8}$ No tenemos más que fijarnos en las cualidades y virtudes que difundían los Manuales medievales como necesarias para ser un buen mercader para entenderlo. El gusto por el orden, la claridad, la precisión...; también el trabajo duro, la discreción, la lealtad, la honorabilidad o la prudencia se ensalzan continuamente. El profesionalismo se entendía ya como la prestación oportuna de servicios de calidad basados en la experiencia, el conocimiento y la prudencia. Ver al respecto mi trabajo «Algunos aspectos de los Manuales de Mercaduría. El valor del aprendizaje. La pereza es llave de la pobreza». Anuario de Estudios Medievales, vol. 41, núm. 2 (2011), pp. 803-817.

${ }^{9}$ En 1397, los conocimientos contables y una buena escritura constituían los fundamentos de la carta de presentación para el muchacho que la compañía Datini enviaba a Valencia. Cien años después, se seguían valorando estas mismas habilidades. El mercader de Burgos Andrés de Melgosa se quejaba de la ineptitud de su factor, Francisco de Atienza, reprochándole que escribiese mal, fallo imperdonable en un factor. B. Caunedo del Potro y M. Sánchez Martín, " Claúsulas comerciales», p. 205. 
ción demandada por los nuevos hombres de negocios ${ }^{10}$. Estos textos, considerados como descendientes del Liber Abbaci de Leonardo da Pisa ${ }^{11}$, se multiplicaron a buen ritmo, siendo más de 300 los manuscritos conservados en Italia anteriores a $1500^{12}$. Todos muy similares, responden a una misma exigencia y se estructuran de igual forma. Utilizan números indoarábigos y comienzan presentándonoslos. A continuación se describe el sistema de numeración posicional en base $10 \mathrm{y}$ las operaciones y reglas esenciales: suma, resta, multiplicación, división, regla de tres. Concluyen insertando una serie más o menos larga de problemas resueltos. En estas obras, aparentemente claras y sencillas, se eliminan las citas y cualquier disgregación teórica para centrarse en lo que se cree que realmente interesaba al mercader, al hombre de negocios y a todo aquél que quisiera aprender la nueva aritmética. Tras el Liber Abbaci, todavía redactado en latín, todos van a estar escritos en lenguas vernáculas. Razones de comodidad e inteligibilidad lo aconsejaban si se quería garantizar su uso por un público que ya no conocía el latín.

Actualmente, el exclusivo protagonismo de Leonardo Pisano, Fibonacci, como precursor de la difusión de las aritméticas mercantiles que prosperaron en las zonas comerciales más desarrolladas del Mediterráneo desde el siglo XIII está

${ }_{10}$ Un panorama general sobre las escuelas y maestros de ábaco nos lo ofrece R. FranCI y L.T. Rigatelli, Introduzioni all Aritmetica Mercantile del medievo e del Rinascimiento. Urbino, Quatro Venti, 1982, especialmente pp. 25-27, y un programa específico del funcionamiento de las más prestigiosas, R. Goldthwaite, «Schools and Teachers of Commercial Arithmetic in Renaissance Florence». Journal of European Economic History, vol. 1 (1972), pp. 418-433. E. Ulivi, «Maestri e scuoie d'abaco a Firenze. La bottega di Santa Trinita». Bollettino di storia delle scienze matematiche, vol. 24 (2004) pp. 43-91.

11 En 1202, Leonardo da Pisa, Fibonacci (1170-1240), publica en lengua latina su Liber Abacci. En el mismo intentó mostrar las ventajas del sistema decimal y de las cifras hindúes sobre el sistema romano y su numeración. Está estructurado en 15 capítulos. Del i al viI, presenta las cifras hindúes y las operaciones elementales con números enteros y fraccionarios, y del viII al final plantea y resuelve problemas de muy diversa índole: de sociedades, de cambio, de aleaciones, conversiones monetarias... Su figura es estudiada y ensalzada por G. SARTON, Introduction to the History of Science, II-II. Baltimore, Williams \& Wilkins, 1927-1948, pp. 611-613 (repr. Nueva York, 1975). También por todas las historias de las matemáticas generales, por ej., C.B. Boyer, Historia de las matemáticas. Madrid, 1986, Alianza, pp. 326-329, quien se lamenta de la inexistencia de versiones latinas accesibles de la obra y remite al trabajo de B. Boncompagni, Scritti di Leonardo Pisano, I (Liber Abacci), II Practica geometriae, Opera Minora. Roma, 1857, p. 62. Sobre su obra y época, M. Morelli y M. TANGheroni, Leonardo Fibonacci. Il tempo, le opere, l'ereditá scientifica. Pisa, IBM, 1994. Recientemente está profundamente estudiado por J. Hoyrup. Remitimos a uno de sus últimos trabajos, que contienen, además, una amplia bibliografía. J. Hoyrup, «Fibonacci-Protagonist or Witness? Who Taught Catholic Christian Europe about Mediterranean Commercial Arithmetic?». Journal of Transcultural Medieval Studies, vol. 1, núm. 2 (2014), pp. 219-247 (en adelante "Fibonacci-Protagonist or Witness?»).

12 W. Van Egmond, «How algebra came to France», en C. Hay (dir.), Mathematics from Manuscript to Print (1300-1600), Oxford, Clarendon Press, 1988, pp. 127-143; encontramos esta cifra en la p. 129. La gran mayoría se describen en otro trabajo del mismo autor, el importante catálogo Practical Mathematics in the Italian Renaissance; a Catalogue of Italian Abbacus Manuscripts and Printed Books to 1600, Istituto e Museo di Storia della Scienza, Monografia 4, Florencia, 1980. Después de la publicación del mismo se identificaron, según el autor, una docena más. 
siendo cuestionado en profundidad por J. Hoyrup ${ }^{13}$. Estando totalmente de acuerdo con otros grandes investigadores ${ }^{14}$ del tema sobre el aprendizaje y familiarización de Leonardo con la aritmética comercial árabe y bizantina ${ }^{15}$, no lo está en que él y su Liber Abbaci hayan sido la primera y única vía de transmisión de esta nueva aritmética. Pone de manifiesto, con sólidos argumentos que tienen en cuenta tanto afirmaciones del propio Fibonacci como escritos similares de las regiones ibérica y provenzal, que la "cultura del ábaco», término acuñado por el propio Hoyrup, surgió en un amplio proceso de interacción con tradiciones árabes dentro de un espacio abierto en torno a la región ibérico-provenzal. Este investigador, además de instarnos a prestar atención a posibles nuevos descubrimientos de manuscritos inéditos, resalta afirmaciones del propio Leonardo narrándonos cómo había aprendido el uso de la numeración indoarábiga también en Provenza o cómo algunos capítulos de su Liber Abbaci se inspiraron en un «magister» castellano ${ }^{16}$. A continuación se adentra en un riguroso estudio comparativo de los diferentes Liber Abbaci editados. Desgrana algunos de los problemas, así como el modo de resolución ${ }^{17}$, y concluye su trabajo con la demostración de la enorme importancia de la tradición ibérica provenzal, no valorada suficientemente, en la difusión de la aritmética comercial. Con esta afirmación estamos totalmente de acuerdo. No podía ser de otro modo, ya que tuvimos la enorme fortuna de localizar y estudiar El Arte del Alguarismo ${ }^{18}$, primer ejemplo conocido hasta el momento de aritmética mercantil castellana. Prescindiendo incluso de este hallazgo, el propio sentido común nos anima a ello. No podemos olvidar que nuestro país se benefició de una fuente, valiosa y directa, que se debe tener en cuenta para explicar la existencia y circulación de este tipo de literatura técnica y que fue su riquísima tradición andalusí. Una tradición que permitió a la sociedad castellana utilizar, cuando esa sociedad lo reclamó, todo el acervo cultural matemático que poseía y conocía

13 En uno de sus últimos trabajos al respecto, «Fibonacci-Protagonist or Witness?», vuelve a insistir en esta idea que ya había desarrollado con anterioridad.

${ }^{14}$ Se refiere como grandes difusores de la misma a los muy respetados y admirados por él mismo y por toda la comunidad de historiadores Elisabetta Ulivi y Van Egmond, J. Hoyrup, «Fibonacci-Protagonist or Witness?», p. 221.

${ }^{15}$ No podía ser de otro modo, pues en la introducción del Liber Abacci el propio Leonardo relata su aprendizaje en Bujara, lugar al que se había trasladado acompañando a su padre, funcionario pisano al servicio de grandes mercaderes italianos. Dato muy conocido que podemos seguir entre otros muchos trabajos, en el de R.E. GRImM, «The autobiography of Leonardo Pisano». Fibonacci Quaterly, vol. 21 (1976), pp. 99-104. Recientemente, el propio Hoyrup ha elaborado la voz Fibonacci para la enciclopedia del islam, donde de modo sucinto recoge su biografía. J. HoyRUP, The Encyclopaedia of Islam, Three, vol. 4, Leiden-Boston, Brill, 2016.

16 J. Hoy Rup, «Fibonacci-Protagonist or Witness?», pp. 124-25.

${ }_{17}$ La dinámica del trabajo es este análisis comparativo, ibidem, p. 226 y ss. Además de analizar los problemas y el método de resolución se detiene en sus enunciados y en la utilización de frases establecidas para presentarlos, ver también pp. 234-235.

18 B. Caunedo del Potro y R. Córdoba de la Llave, El arte del alguarismo. Un libro castellano de aritmética comercial y de ensayo de moneda. Estudio, edición, glosario e indices. Salamanca, Junta de Castilla y León, 2000. 
desde años atrás y que la propia península podría poner a disposición del Occidente europeo ${ }^{19}$, incluida la propia Italia ${ }^{20}$.

Un magnífico ejemplo de ese rico acervo aritmético lo constituye el Liber Mahameleth, obra escrita en Castilla, en latín, a mediados del siglo xir y que utiliza en su título el término Mahameleth, versión latinizada de la palabra que significa "matemáticas de negocios». Esta obra, que ha sido profundamente estudiada por el profesor Jacques Sesiano a lo largo de más de 40 ańos, cuenta gracias a su intenso y fructífero trabajo con una excelente y reciente edición crítica ${ }^{21}$. Consta la obra original de dos partes, una teórica y otra de aplicaciones prácticas. La primera trata de la teoría de las proporciones y de las operaciones aritméticas fundamentales: suma, resta, multiplicación, división y raíz cuadrada. También se encuentran referencias a la resolución de ecuaciones de primero y segundo grado. Menciona a Euclides, Arquímedes, Nicómaco de Gerasa, Al- Khowarizmi y Abu Kamil, autoridades que corresponden a lo que podría esperarse fuera conocido en Castilla en esos años del siglo Xiı, ya que la obra estaría destinada al uso de la comunidad cristiana de Sevilla, desde donde se trasladaría a Toledo. En este lugar aparece Domingo Gundisalvo como uno de sus primeros lectores. En su obra De

19 Si nos centramos en la Península Ibérica, esta labor se desarrollaría fundamentalmente en los siglos XI y XII, cuando acudieron pensadores de diferentes lugares con el afán de traducir del árabe al latín. Las obras científicas supusieron un $68 \%$ de todo lo traducido y, dentro de éstas, el 48\% fueron obras de matemáticas. J. Lomba Fuentes, "Aportaciones musulmanas a la renovación filosófica del siglo xII», en XXIV Semana de Estudios Medievales, Renovación intelectual del Occidente Europeo, Pamplona, Gobierno de Navarra, 1998, pp. 135-167.

${ }^{20}$ Un posible origen hispano atribuye J. Hoyrup al considerado primer tratado de álgebra en lengua vulgar, Tractatus Algorismi, de Jacopo de Firenze. J. Hoy Rup, «Vat. Lat. 4826 Jacopo de Firenze. Tractatus algorismi. Preliminary transcription of the manuscript with ocasional commentaries». Filosofi og Videnskabsteori Pa Roskilde Universitetscenter, vol. 3.b (1993), pp. 1-114, y "Jacopo de Firenze and the beggining of Italian vernacular Algebra». Filosofi og Videnskabsteori Pa Roskilde Universitetscenter, vol. 6 (2003), pp. 1-35.

${ }^{21}$ J. Sesiano, The Liber Mahameleth: A 12th-century Mathematical Treatise. Springer, 2014. Esta magnífica edición consta de tres volúmenes. El primero contiene una introducción general que describe los diversos manuscritos existentes (cuatro), el contenido de la obra y lo que sabemos de su autor, Juan de Sevilla. Cree que el libro estaría destinado al uso de la comunidad cristiana de Sevilla y que de allí se trasladó a Toledo. En el segundo volumen efectúa una traducción al inglés y elabora un glosario de términos latinos, importante para el conocimiento de los términos científicos latinos en ese momento. Por último, en el tercero, analiza los problemas con un resumen del procedimiento matemático involucrado en cada capítulo. Esta obra es el colofón al trabajo iniciado en torno a 1968 cuando en el Primer Coloquio Internacional de Historia de las matemáticas árabes, Sesiano nos ofreció su primera aproximación al mismo. "Le liber Mahameleth, un traité mathématique latin composé au XII siécle en Espagne», en Histoire des Mathematiques arabes, Premier Colloque International sur l'Histoire des Mathematiques arabes, Alger, 1968, pp. 69-70, no nos dice nada del autor más que su localización en Castilla en la segunda mitad del siglo XII. Poco después lo identifica, aunque con algunas reservas, con Juan Hispalensis y adelanta en unos años la fecha de elaboración, 1140, 11351153, "Der Liber Mahameleth", 8, XVIII International Congress of History of Science, HamburgoMúnich, 1989. La obra cuenta también con otra edición crítica realizada por A.M. VlasschaerT (ed.), Le Liber Mahameleth: Édition critique et commentaires. Stuttgart, Franz Steiner Verlag, 2010. En este trabajo no se analizan los problemas, ya que tiene un carácter filológico. 
Divisione Philosophiae incluye algunas precisiones aritméticas, especificando que éstas se tratan en otro trabajo llamado «mahameleth $»^{22}$.

La segunda parte la componen una colección de problemas, aplicaciones de la matemática a la vida diaria, sobre compra y venta de mercancías, contratación de obreros, distribución y preparación de alimentos, cambios de moneda y pequeños ejercicios de geometría ${ }^{23}$.

A pesar de su enorme importancia, la obra no tuvo notoriedad en su momento y pasó casi inadvertida en los años e incluso siglos posteriores salvo los ejemplos ya comentados. Las razones las encuentra Sesiano en el mismo contenido de la obra, que él califica a la vez de decepcionante, fascinante y desconcertante. Decepcionante para quien esperase encontrar en ella novedades, innovaciones, riqueza y diversidad que no contiene. Fascinante porque es uno de los documentos aritméticos más antiguos de Europa Occidental que está inspirado directamente en fuentes árabes. Y desconcertante por su ambivalencia. Por su naturaleza práctica parecía estar orientada a todos aquéllos que se dedicaban al comercio, administración o gestión, pero éstos no disponían de los conocimientos necesarios como para entender unos cálculos en ocasiones complejos. ¿Qué mercader estaría preparado para comprender Los Elementos de Euclides antes de acometer la lectura del Liber Mahameleth? Si a éstos les superaba el elevado nivel matemático de la obra, a los matemáticos les desagradaba el número de problemas prácticos.

No tuvo, pues, la acogida alcanzada por el mencionado Liber Abacci, de Leonardo Pisano, acérrimo defensor del sistema indoarábigo. La publicación de una segunda edición de su manual, en 1227, iniciará una ardua contienda entre el tradicional método de cálculo, el ábaco, y el nuevo, el algorismo, que se irá imponiendo lentamente y que incluso trascenderá a bellas representaciones iconográficas que impregnarán una época ${ }^{24}$. Esta lucha es en realidad la mantenida entre los partidarios de continuar utilizando la numeración romana y el artilugio necesario para realizar los cálculos con esa numeración, llamados «abacistas», y los que pre-

${ }^{22}$ En la edición de 2014, Sesiano especifica también cómo el dominico francés Vincent de Beauvines (1264) repite lo que loa Domingo Gundisalvo y recoge nuevamente el término mahameleth. También en el mismo siglo XIII se conoció en Italia (la mejor copia que se conserva en la actualidad es una italiana del siglo XIv).

${ }^{23}$ J. Sesiano, «Survivance médiévale en Hispanie d'un problème né en Mesópotamie». Centaurus, vol. 30 (1980), pp. 18-61. Tras una reflexión sobre la dificultad de la transmisión de conocimientos, hace una historia de los denominados problemas de escalera. El pie de una escalera apoyado contra un muro que tiene su misma altura se va desplazando; en consecuencia, también la cima de la escalera se desplazará. Se trata de determinar una longitud conociendo dos. Se aplica propiamente a una escalera y muro, a un árbol roto y caído, "problemas del árbol roto» o "problemas de las dos torres», cuando tratan de buscar un punto en el suelo que esté a igual distancia que las cimas de las torres. El autor va localizando estos tres tipos de problemas en diferentes autores medievales: al-Biruni, A'braham ibn Ezra o Leonardo de Pisa.

${ }^{24}$ Un buen ejemplo es «Margarita Philosophica» (1503), de Gregor Reisch, representación que aparece reproducida en múltiples trabajos. Así, en M.A. OuAKIn, El misterio de las cifras. Barcelona, Ma Non Troppo, 2006, p. 118. El autor toma partido claramente por la numeración indoarábiga. 
ferían optar por la novedosa numeración indoarábiga deslumbrados, como Fibonacci, por sus clarísimas ventajas operativas. Éstos fueron los algoristas. Frente a ellos se argumentó el peso de la tradición, la desconfianza, el temor al fraude o las ansias de unas mayores garantías de autenticidad.

Creemos que el mayor recelo vino del uso del 0 , al que no se trató de la misma forma que al resto de los números, y del complicado sistema posicional en base 10. Resultaba muy difícil de entender que hay dos usos para el cero, ambos muy importantes, pero distintos. En primer lugar, significa la ausencia de una cifra en un sistema numeral por posición y, en segundo, supone el concepto de cantidad nula, es un número en sí mismo. Hizo falta mucho tiempo para darse cuenta de estos dos usos, una de las más grandes aportaciones de los hindúes pero complejas y difíciles de entender. De hecho, casi todos los manuales de ábaco llaman especialmente la atención al lector cuando explican este doble uso del 0 con signos de admiración o advertencia ${ }^{25}$. Además, los que no lo entendían aducían que ese extraño símbolo 0 fácilmente podía convertirse en su representación gráfica en un 6 o en un 9. También resultaba difícil de explicar y entender cómo la cantidad representada por un dígito está determinada por su posición, y cómo esos valores de posición se incrementaban de derecha a izquierda en potencias de $10^{26}$. La adición de un numeral al final de cualquier número crea otro más grande. No gustaba. No lo comprendían.

Dificultad sí, desconfianza también. Ambas desempeñaron un papel importante en reforzar la inercia y el conservadurismo que retrasó la introducción del nuevo sistema indoarábigo. No obstante, a ritmo lento y con importantes diferencias entre unos países y otros, Italia y Alemania, por ejemplo ${ }^{27}$, el triunfo de los abacistas lo determinarían las necesidades y exigencias contables que el incremento y mejora del comercio estaba generando. Efectivamente, además de sus indudables ventajas operativas, observamos en múltiples facetas un protagonismo tras-

${ }_{25}$ Así, por ejemplo, El Arte del Alguarismo, p. 69, comienza presentando los números del 1 al 9, a los que el autor llama letras del alguarismo, mientras que al 0 le llama Çifra. A continuación hace notar que esta cifra por sí sola no vale nada, pero al poner uno o varios símbolos delante adquiere un elevado valor.

${ }_{26}$ En El Arte del Alguarismo, pp. 134-135, el valor de cada cifra que depende de su posición es nombrado como presçio de lo que valen las letras.

27 E. Hernández Esteve, «Incunables de Aritmética comercial anteriores a la Summa de Luca Paccioli», en II International Conference «Before and after Luca Pacioli», SansepolcroPerugia-Perugia-Florence, 2011, pp. 6-7, nos la explica con múltiples detalles. Destaca cómo el Statuto dell'Arte di Cambio emitido por el Ayuntamiento de Florencia en 1299, cuando el uso de la numeración indoarábiga estaba extentido entre los mercaderes italianos, prohibió su empleo en los libros de cuentas de los banqueros residentes en el municipio. Las autoridades venecianas siguieron su ejemplo ese mismo año, y en 1348 la Universidad de Padua dispuso que el catálogo de sus libros llevara los precios escritos en números romanos. Y cómo en Alemania la oposición al nuevo sistema de numeración se prolongó bastante más en el tiempo. A finales del siglo Xv, unas ordenanzas municipales de Fráncfort dictaminaban que los maestros de cálculo no operasen con números indoarábigos. Era tal el arraigo de los números romanos en este país en el siglo xvi que a los números romanos se les llamaba también «números alemanes». 
cendental del nuevo sistema que aportaría un conjunto de técnicas para aplicar a la resolución de problemas económicos. El manejo de datos, la capacidad de procesarlos y relacionarlos, también ayudó a revelar una información significativa, verdadera y concreta, de carácter numérico, y que sirvió para la toma de decisiones acertadas. Además de estos beneficios, queremos destacar cómo avaló el nacimiento de una nueva disciplina, la contabilidad por partida doble, o cómo se bautizaron algunas conocidas formas de operar con claras denominaciones mercantiles.

\section{UNA NUEVA DISCIPLINA}

La contabilidad moderna, que no se entiende sin el nuevo sistema ${ }^{28}$, es una contabilidad por partida doble. Cuando Luca Pacioli publica en Venecia en 1494 su obra Summa de Arithmetica geometría proportioni et proportionalita y nos la explica ${ }^{29}$, lo hace utilizando los novedosos métodos de cálculo que la nueva aritmética permitía. El fraile italiano, hoy reconocido como padre de la contabilidad moderna, explicó con claridad la contabilidad de doble entrada que ya venía utilizándose en Italia desde el siglo XIV, convirtiéndola en contabilidad por partida doble o veneciana; esto es, abonos y cargos, activos y pasivos, el haber y el debe. En 36 capítulos nos detalla con amplitud, precisión y coherencia múltiples aspectos de la nueva disciplina: la técnica de la partida doble; la naturaleza y forma de funcionamiento de los tres libros principales: Borrador, Diario y Mayor; el sentido y razón de ser de la contabilidad; las reglas prácticas de anotar el patrimonio y las operaciones comerciales comenzando por la clasificación, valoración y registro de los bienes poseídos; el modo de cerrar y traspasar las cuentas y los libros y de hacer el balance de comprobación ${ }^{30}$. Claramente se aprecia cómo la contabilidad proporcionaba nuevas herramientas para el planteamiento y el control de la organización.

${ }^{28}$ E. Hernández Esteve, «La contabilidad y la numeración arábiga». AECA, Revista de la Asociación Española de Contabilidady Administración de Empresas, vol. extra 76 (2006), pp. 43-48. Nos relata cómo algunos historiadores no podían concebir que la partida doble pudiera haberse originado sin conocimiento de la nueva numeración.

${ }^{29}$ E. Hernández Esteve ha realizado un excelente trabajo, estudio introductorio, traducción y notas, del título noveno, tratado xi de la Summa, que se refiere a la contabilidad, Tractatus XI Particularis de Computis et Scripturis: Luca Pacioli: De las cuentas y las escrituras. Madrid, Asociación Española de Contabilidad y Administración de Empresas (AECA), 1994. Sigo esta primera edición, aunque en el 2009 se publicó una segunda edición.

${ }^{30}$ Luca Pacioli. Seguimos fielmente lo resaltado por Hernández Esteve, pp. 72-80. 


\section{REGLA DE LA COMPAÑÍA}

Pero la nueva aritmética no sólo sirvió de soporte para una nueva disciplina, sino que sus ensalzadas ventajas operativas permitieron bautizar, no sólo las cifras ${ }^{31}$ sino también algunas formas de operar con claras denominaciones mercantiles. Así, por ejemplo, la regla de tres, denominada la regla de oro de los mercaderes ${ }^{32}$ y la regla de la compañía, reglas que se aplicaron a uno de los principales instrumentos de gestión del gran comercio, la compañía. Recordemos que esta asociación fue fruto de la gradual evolución de los derechos de propiedad, de los acuerdos contractuales, así como de la voluntad de concentrar capital o repartir riesgos.

La regla de la compañía es en realidad un reparto proporcional, en el que la cantidad a repartir son los beneficios o pérdidas de una sociedad. Su denominación, el triunfo del término ${ }^{33}$, nos revela su estrecha vinculación con el mundo de los negocios. En un primer momento, los problemas sobre la adjudicación de beneficios que se incluían en los manuales de aritmética mercantil no se distinguían de otros ejercicios sobre reparto proporcional que se presentaban en los mismos y que se resolvían con procedimientos de regla de tres. Si nos fijásemos exclusivamente en manuscritos peninsulares medievales veríamos que así ocurre, por ejemplo, en El Arte del Alguarismo ${ }^{34}$, manuscrito castellano de finales del siglo XIV, en el que no existe ningún apartado titulado regla de la compañía pero sí presenta y resuelve problemas similares ${ }^{35}$. Tendríamos que esperar a 1482 para que Francesc de Santcliment, en su Summa de lárt Aritmética ${ }^{36}$, escrita en catalán, utilizase esta expresión y además la justificase. Lo hace explicándonos que en esta clase de

31 E. Hernández Esteve, «Incunables de Aritmética comercial», p. 7, relata cómo los números árabes se llamaron «figure mercantesche», cifras mercantiles.

32 Realmente la utilizaron muchísimo porque les permitía resolver cantidad de problemas y les resultaba fascinante que con tres términos conocidos se obtuviese un cuarto desconocido. El Arte del Alguarismo contiene un total de 192 ejercicios. De éstos, 28, un 14,58\%, se resuelven utilizando la regla de tres. Es el procedimiento más utilizado tras las operaciones elementales ( 52 , $27 \%)$ y las fracciones $(67,34,89 \%)$, p. 75.

33 Como señala J. Navarro Loidi, «La regla de la Compañía y la didáctica del reparto proporcional». Sigma: Revista de Matemáticas = Matematika aldizkaria, vol. 28 (2006), pp. 117130, la regla de la compañía ha sido durante muchos siglos un apartado fijo de los manuales de aritmética publicados en Espańa, aunque recientemente se ha dejado de incluir en los mismos.

34 B. Caunedo del Potro y R. Córdoba de la Llave, El Arte del Alguarismo.

35 Por ej., «Fas esta cuenta, 3 compañeros an en compañía 100 libras y el uno avía el medio y el otro el $1 / 3$ y el otro el $1 / 4$, demando ¿quántos vernán a cada uno por su derecha regla? Acuérdate que deves desir, $1 / 2,1 / 3,1 / 4$. se falla en 12 , que el $1 / 2$ es 6 y el $1 / 3$ es 4 y el $1 / 4$ es 3 , otrosy deves dezir 6 e 4 e 3 son 13 e di, otrosy, sy 13 fuesen 100 ¿qué serán 6?». Es el problema 69, p. 170 de la obra presentada en la nota anterior.

36 A. Malet (ed.), Summa de lárt Aritmética de Francesc Santcliment. Vich, Eumo, 1998 (en adelante Summa de lárt Aritmética). Aparece también en muchos de los italianos, también en el que está al frente de la producción, el Liber Abaci, de Leonardo de Pisa. 
cálculo son necesarias tres cosas: las partes, el tiempo y la pérdida o ganancia ${ }^{37}$, señalando que es esa posibilidad, la de ganar o perder, la que determina el nombre de la regla. Más adelante reconoce claramente que los problemas de compañías son muy semejantes a los de la regla de tres, siendo la única diferencia que en este último tipo de problemas no hay comúnmente más de tres partes, mientras que en los de las compañías se encuentran a menudo más partes ${ }^{38}$.

Unos años después, ya en los albores del siglo XVI, en 1515, el clérigo zaragozano mosén Juan de Andrés en su Manual de Aritmética Mercantil ${ }^{39}$ es todavía más explícito, y cuando realiza la presentación de la regla, la vincula directamente con el mundo del comercio, y así nos dirá que «... trata de la operación que se faze entre dos o tres mercaderes y dende arriba en el arte mercantivol quando fazen compañía y ponen su canal diferentemente...» para continuar un poco más adelante: «... y cómo debe ser la ganancia o la perdida partido entre tales mercaderes según su canal de cada uno proporcionalmente...», definiciones que animan a pensar que el cálculo elemental que los mercaderes aprendían tenía como uno de sus objetivos la evaluación y cuantificación de sus ingresos, gastos o beneficios. Normalmente siempre se contemplan ganancias y éstas han de ser proporcionales al capital aportado por cada socio y al tiempo de inversión de cada capital, en cuyo caso se denomina "con tiempo".

Contiene la colección de mosén Juan de Andrés 37 problemas relativos a la regla de la compañía. Éstos presentan muy diversos matices, tanto en las aportaciones como en la diversidad de modalidades de reparto, lo que hace que cada problema aparezca como una situación totalmente nueva. Queremos destacar aquéllos cuyos enunciados evidencian unas técnicas y prácticas mercantiles novedosas. Así, por ejemplo, resulta evidente que una de las obligaciones esenciales de los socios fue la aportación de capital que hiciese posible el establecimiento y el comienzo de la sociedad. Estas contribuciones podían ser de naturaleza diversa, ya que los aportes monetarios -los más frecuentes- se combinaron con el trabajo y cometido

${ }^{37}$ A. Malet (ed.), Summa de lárt Aritmética. «Les quals 3 coses són les parts; la segona és lo temps; la terça és lo guany o pérdua. Car per ço se diuen companyes, que així es passen a rise de perdre com de guanyar", p. 248.

${ }_{38}$ Ibidem, «...que la diferencia que és entre les companyes i la regla de tres no és sinó per quant en les companyies se troben moltes vegades moltes parts, per ésser molts en la companyia, $i$ en la regla de tres no hi ve sinó comunaament tres parts. I aquesta és la diferencia entre les dites regles. És veritar que qualsevol raó de companyies per la dita regla de tres se pot fe...r», p. 249.

${ }^{39}$ He estudiado parte del contenido de esta obra en «Un manual de Aritmética mercantil de Mosén Juan de Andrés». Pecunia: Revista de la Facultad de Ciencias Económicas y Empresariales, vol. 8 (2009), pp. 71-96, y en concreto los problemas de la regla de compañía en mi trabajo "Otros datos sobre las compañías castellanas en la Baja Edad Media», en M.I. del Val Valdivieso y P. Martínez Sopena (coords.), Castilla y el Mundo Feudal: Homenaje al profesor Julio Valdeón, Salamanca, Junta de Castilla y León, 2009, pp. 625-638. Con posterioridad Elena Ausejo nos ofreció más datos sobre su autor, E. Ausejo Martínez, «Nuevos datos sobre Mosén Juan de Andrés, autor de 'Sumario Breve D’la Pratica dela Arithmetica' (1515)», en F.A. González Redondo (coord.), Ciencia y Técnica entre la paz y la guerra: 1714, 1814, 1914, Sociedad Española de Historia de las Ciencias y de las Técnicas, Sencyt, i, 2016, pp. 457-463. 
personal de alguno de los socios en la firma o con la entrega de objetos de valor que, como una joya ${ }^{40}$, pudiesen ser valorados en términos económicos. También se pueden obtener datos sobre los beneficios de las compañías en los que se evidencia de forma inexorable la importancia del factor tiempo en las operaciones mercantiles. Resulta de extraordinario interés la nueva valoración del tiempo que estos ejercicios reflejan. Tras leer su enunciado, no se puede dudar que ya entonces el grupo mercantil creía firmemente que en cualquier operación con capital su rentabilidad dependía del tiempo ${ }^{41}$. Tampoco dudarían de la conveniencia de incentivar el trabajo de un buen factor, imprescindible para un correcto funcionamiento de la firma. Su dedicación y esfuerzo podía ser considerado una inversión en las compañías, también abiertas a aceptar diversas incidencias en las aportaciones de capital. Así, se contemplan aportaciones extras de los mismos socios realizadas con posterioridad a la firma del contrato de constitución de la sociedad o la incorporación de un nuevo mercader o de un simple inversor a la misma cuando ya estaban establecidas las aportaciones y ganancias de cada uno de los socios. Resulta bastante evidente que el capital de una compañía podía incrementarse durante la duración de la misma pues estas sociedades comerciales permitieron múltiples combinaciones de reagrupamiento de socios o de aportaciones de fondos diversos por tiempos también diferentes ${ }^{42}$. El autor de este manual manifiesta de forma reiterada la movilidad y divisibilidad de la inversión. Para él, las operaciones de compra y de venta o las inversiones no son algo estático que se realiza una vez y se completa, sino que son algo dinámico en el tiempo y que pueden cambiar varias veces en el transcurso del mismo.

Hemos visto cómo el mercader estaba profesionalizando su oficio y su formación, pero en su horizonte le faltaba otra meta por alcanzar: su honorabilidad y total integración social. El mercader de éxito también debería mostrar una conducta ejemplar, una trayectoria de responsabilidad y ética en el desarrollo de su actividad y funciones. Un nuevo reto que en estas líneas no podemos abordar, aunque sí anticipar que también lo lograría. Más tarde que estos manuales de aritmética a los que nos hemos referido, irían apareciendo otros que les proporciona-

40 B. Caunedo, «Un Manual de Aritmética», p. 87.

${ }^{41}$ Ibidem, p. 88.

${ }^{42}$ Los enunciados de los problemas recogen ejemplos de disolución de las compañías antes del fin del plazo previsto (el mencionado de uno a tres ańos), pero no analizan los motivos. Podemos pensar que irían desde el deseo de los socios de poner fin a la relación o a causas de fuerza mayor como el fallecimiento de alguno de ellos. Éstas son las causas que se describen en algunas compañías como "condiciones propias de funcionamiento", ver mi trabajo "Compañías mercantiles castellanas a fines de la Edad Media». Medievalismo, vol. 3 (1993), pp. 39-57, donde también describo diferentes tipos de compañías, pp. 42-49. 
rían valores, normas, códigos de conducta, costumbres... para lograr que su actuación no fuese deleznable, sino ejemplar y decorosa ${ }^{43}$.

Recepción: junio 2017, aceptación: noviembre 2017

${ }^{43}$ Los primeros ejemplos y mejor conocidos serán los italianos. Destacamos la obra de B. Cotrugli, Della Mercatura et del Mercante Perfetto, estudiado y editado por U. Tucci en Venecia, Arsenale Editrice en 1990, edición ésta con la que hemos trabajado, aunque existe una nueva edición más reciente, de 2016, y una traducción al inglés de 2017: C. Carraro y G. Favero (eds.), Benedetto Cortugli. The Book of the Art of Trade. Contiene capítulos de Niail Ferguson, Giovanni Favero, Mario Infelise, Tiziano Zanato y Vera Ribaudo, trad. John F. Phillimore, Palgrave Macmillan, 2017; datos sobre los mismos en mi trabajo «Algunos aspectos de los Manuales de Mercaduría», pp. 803-817. 


\section{BIBLIOGRAFÍA}

Ausejo Martínez, Elena, «Nuevos datos sobre Mosén Juan de Andrés, autor de “Sumario Breve D’la Pratica dela Arithmetica (1515)”", en Francisco A. González Redondo (coord.), Ciencia y Técnica entre la paz y la guerra: 1714, 1814, 1914, Sociedad Espańola de Historia de las Ciencias y de las Técnicas, Sehcyt, i, 2016, pp. 457-463.

Boncompagni, Baldassarre, Scritti di Leonardo Pisano, I (Liber Abacci), II Practica geometriae, Opera Minora. Roma, 1857.

Carraro, Carlo y Giovanni Favero (eds.), Benedetto Cortugli. The Book of the Art of Trade. Trad. John F. Phillimore, Palgrave Macmillan, 2017.

Cipolla, Carlo Maria, El gobierno de la moneda. Ensayos de historia monetaria. Barcelona, Crítica, 1994.

Casado Alonso, Hilario, "Comercio y Nacimiento del Estado Moderno en Castilla (ss. XIV-Xvi). Algunas reflexiones a la luz de nuevas corrientes de investigación internacional», en $E l$ Estado en la Baja Edad Media. Nuevas perspectivas metodológicas, Zaragoza, Universidad de Zaragoza, 1999, pp. 51-75.

Castillo Gómez, Antonio, Escrituras y Escribientes. Práctica de la cultura escrita en una ciudad del Renacimiento. Las Palmas de Gran Canaria, Fundación de Enseñanza Superior a Distancia de Las Palmas de Gran Canaria, 1997.

Caunedo del Potro, Betsabé, «La formación y educación del mercader», en El Comercio en la Edad Media, XVI Semana de Estudios Medievales Nájera y Tricio, 2005, Nájera, Instituto de Estudios Riojanos.

Caunedo del Potro, Betsabé, «Algunos aspectos de los Manuales de Mercaduría. El valor del aprendizaje. La pereza es llave de la pobreza». Anuario de Estudios Medievales, vol. 41, núm. 2 (2011), pp. 803-817.

Caunedo del Potro, Betsabé, «Un manual de Aritmética mercantil de Mosén Juan de Andrés». Pecunia: Revista de la Facultad de Ciencias Económicas y Empresariales, vol. 8 (2009), pp. 71-96.

Caunedo del Potro, Betsabé, "Otros datos sobre las compañías castellanas en la Baja Edad Media», en M. ${ }^{a}$ Isabel del Val Valdivieso y Pascual Martínez Sopena (coords.), Castilla y el Mundo Feudal: Homenaje al profesor Julio Valdeón, Salamanca, Junta de Castilla y León, 2009, pp. 625-638.

Caunedo del Potro, Betsabé y Ricardo Córdoba de la Llave, El arte del alguarismo. Un libro castellano de aritmética comercial y de ensayo de moneda. Estudio, edición, glosario e indices. Salamanca, Junta de Castilla y León, 2000.

Caunedo del Potro, Betsabé y Margarita Sánchez Martín, «Cláusulas comerciales: ¿Acatamiento o trasgresión? El factor de negocios en la Europa Atlántica», en Jesús Á. SolóRzano TElechea, Beatriz Arizaga Bolumburu y Louis Sicking (eds.), Diplomacia y Comercio en la Europa Atlántica Medieval, Logroño, Instituto de Estudios Riojanos, 2015, pp. 187-219.

Cotrugli, Benedetto, Della Mercatura et del Mercante Perfetto. Ed. Ugo Tucci, Venecia, Arsenale Editrice, 1990.

De Roover, Raymond, «The commercial revolution of the thirteen century». Bulletin of the Business Historical Society, vol. xvi (1942), pp. 34-39 
De Roover, Raymond, «The organization of trade», en The Cambridge Economic History of Europe, vol. III, Cambridge, Cambridge University Press, 1965, pp. 42-118.

Franci, Rafaella y Laura Toti Rigatelli, Introduzioni all Aritmetica Mercantile del medievo e del Rinascimiento, Urbino, Quatro Venti, 1982.

Goldthwaite, Richard A., «Schools and Teachers of Commercial Arithmetic in Renaissance Florence». Journal of European Economic History, vol. 1 (1972), pp. 418-433.

Grimm, Richard E., «The autobiography of Leonardo Pisano». Fibonacci Quaterly, vol. 21 (1976), pp. 99-104.

Hernández Esteve, Esteban, «Incunables de Aritmética comercial anteriores a la Summa de Luca Paccioli», II International Conference «Before and after Luca Pacioli», Sansepolcro-Perugia-Perugia-Florence, 2011, pp. 6-7.

Hernández Esteve, Esteban, «La contabilidad y la numeración arábiga». AECA, Revista de la Asociación Española de Contabilidad y Administración de Empresas, vol. extra 76 (2006), pp. 43-48.

Hoyrup, Jean, «Vat. Lat. 4826 Jacopo de Firenze. Tractatus algorismi. Preliminary transcription of the manuscript with ocasional commentaries». Filosofi og Videnskabsteori Pa Roskilde Universitetscenter, vol. 3.b (1993), pp. 1-114.

Hoyrup, Jean, «Jacopo de Firenze and the beggining of Italian vernacular Algebra». Filosofi og Videnskabsteori Pa Roskilde Universitetscenter, vol. 6 (2003), pp. 1-35.

Hoyrup, Jean, «Fibonacci-Protagonist or Witness? Who Taught Catholic Christian Europe about Mediterranean Commercial Arithmetic?». Journal of Transcultural Medieval Studies, vol. 1, núm. 2 (2014), pp. 219-247.

Hoyrup, Jean, The Encyclopaedia of Islam, Three, vol. 4, Leiden-Boston, Brill, 2016.

Lomba Fuentes, Joaquín, "Aportaciones musulmanas a la renovación filosófica del siglo XiI», en XXIV Semana de Estudios Medievales, Renovación intelectual del Occidente Europeo, Pamplona, Gobierno de Navarra, 1998, pp. 135-167.

Luca Pacioli, De las cuentas y las escrituras. Madrid, Asociación Española de Contabilidad y Administración de Empresas (AECA), 1994.

Malet, Antoni (ed.), Summa de lárt Aritmética de Francesc Santcliment. Vich, Eumo, 1998.

Morelli, Marcello y Marco Tangheroni (eds.), Leonardo Fibonacci. Il tempo, le opere, l'ereditá scientifica. Pisa, Pacini, 1994.

Navarro Loidi, Juan, «La regla de la Compañía y la didáctica del reparto proporcional». Sigma: Revista de Matemáticas = Matematika aldizkaria, vol. 28 (2006), pp. 117-130.

Ouakin, Marc Alain, El misterio de las cifras. Barcelona, Ma Non Troppo, 2006.

Sarton, George, Introduction to the History of Science, II-II. Baltimore, Williams \& Wilkins, 19271948, pp. 611-613 (repr. Nueva York, 1975).

Sesiano, Jean, The Liber Mahameleth: A 12th-century Mathematical Treatise. Springer, 2014 [https:// link.springer.com/book/10.1007\%2F978-3-319-03940-4].

SESIANO, Jean, «Le liber Mahameleth, un traité mathématique latin composé au XII siécle en Espagne», en Histoire des Mathematiques arabes, Premier Colloque International sur l'Histoire des Mathematiques arabes, Alger, 1968, pp. 69-70. 
SEsIano, Jean, "Der Liber Mahameleth», en XVIII International Congress of History of Science, 8, Hamburgo-Múnich, 1989.

Sesiano, Jean, «Survivance médiévale en Hispanie d’un problème né en Mesópotamie». Centaurus, vol. 30 (1980), pp. 18-61.

Ulivi, Elisabetta, «Maestri e scuoie d'abaco a Firenze. La bottega di Santa Trinita». Bollettino di storia delle scienze matematiche, vol. 24 (2004), pp. 43-91.

Vlasschaert, Anne Marie (ed.), Le Liber mahameleth: Edition critique et commentaires. Stuttgart, Franz Steiner Verlag, 2010.

VAN Egmond, Warren, «How algebra came to France», en C. Hay (dir.), Mathematics from Manuscript to Print (1300-1600), Oxford, Clarendon Press, 1988, pp. 127-143.

VAn Egmond, Warren, Practical Mathematics in the Italian Renaissance; a Catalogue of Italian Abbacus Manuscripts and Printed Books to 1600. Florencia, Istituto e Museo di Storia della Scienza, Monografia 4, 1980. 